\title{
Knochenersatzmaterialien: Neue Möglichkeiten und Techniken
}

\author{
W. Linhart, N. M. Meenen, J. M. Rueger
}

\section{Zusammenfassung}

Die guten Erfahrungen des letzten Jahrzehnts mit Hydroxylapatitkeramiken zur Auffüllung v.a. metaphysärer Knochendefekte haben die Bedeutung von Knochenersatzmaterialien im klinischen Alltag bestätigt. Auf der Suche nach biologisch abbaubaren Substanzen haben sich in letzter Zeit zunehmend Kalziumphosphatzemente etabliert. Für einige Indikationen, wie langstreckige diaphysäre Defekte oder Situationen mit schlechter Weichteildeckung sind die Möglichkeiten des Knochenersatzes noch immer sehr begrenzt. Die vorliegende Arbeit zeigt verschiedene Indikationen für die einzelnen Materialien auf und gibt einen Ausblick auf die zu erwartende Entwicklung im Bereich des Knochenersatzes und des Tissue Engineering.

\section{Einleitung}

Knöcherne Defekte entstehen durch Trauma oder Tumor, seltener durch Osteitis. Es kommt zum Verlust von Knochenteilen (z.B. bei offenen Frakturen) oder zum Strukturverlust von Knochensubstanz (z. B. bei Impressionsfrakturen). Da die mechanische Belastbarkeit des Knochens eine zentrale Bedeutung für die ungestörte Beanspruchung des entsprechenden Skelettabschnittes besitzt, ist die möglichst frühzeitige Stabilisierung und Wiederherstellung der mechanischen Funktion des geschädigten Areals von herausragender Bedeutung, um Auswirkungen des Knochendefektes auf die Lebensführung eines Patienten zu minimieren. Als Negativfolgen von Knochendefekten können z.B. Querschnittssyndrome nach Wirbelkörpersinterung, Gelenkzerstörung nach intraartikulären Impressions- oder Defektfrakturen, Verkürzungen oder Pseudarthrosen nach diaphysären Defekten genannt werden.

Die positiven Erfahrungen der letzten Jahre mit keramischen Knochenersatzstoffen haben die Bedeutung von Knochenersatzmaterialien im klinischen All-

OP-JOURNAL 2000; 16: 294-298

(c) Georg Thieme Verlag Stuttgart · New York tag bestätigt $[5,8,9]$. Für viele Indikationen in der Orthopädie und der Unfallchirurgie gilt allerdings weiterhin die autogene Spongiosaplastik bzw. das autogene Knochentransplantat als goldener Standard zur Auffüllung oder Überbrückung knöcherner Defekte. Die Vorteile der autogenen Transplantate gegenüber den Knochenersatzmaterialien liegen in den unübertroffenen biologischen Eigenschaften des körpereigenen Materials und für bestimmte Indikationen sehr guten biomechanischen Eigenschaften (z. B. trikortikaler Beckenspan). Zu den Situationen, in denen autogene Transplantate daher weiterhin große Bedeutung haben, zählen Pseudarthrosen, zu deren Ausheilung osteoinduktive Eigenschaften des Transplantates gefordert sind. Sowie Defekte in diaphysären Knochensegmenten aufgrund der komplexen mechanischen Beanspruchung und knöcherne Substanzverluste durch Infekte und bei schlechter Weichteildeckung bei denen sich die Applikation von Fremdmaterial verbietet.

Eine Vielzahl von Nachteilen autogener Knochentransplantation sind jedoch bekannt. Zunächst muss dem Patienten zur Entnahme eine Erweiterung der Operation mit Verlängerung der OP-Zeit und der Narkosedauer zugemutet werden. Auch sind im Bereich der Entnahmestellen auftretende Komplikationen wie aus- geprägte Hämatombildung, Infektionen, Verletzung des N. cutaneus femoris lateralis, Beckenschaufelfrakturen und anhaltende Schmerzen ausreichend dokumentiert und werden in der Literatur mit bis zu $70 \%$ angegeben $[11,13]$.

Als Alternative zur autogenen Transplantation wurde aus naheliegenden Überlegungen über viele Jahre die allogene Transplantation von kryokonserviertem Knochen durchgeführt, nachdem sich in experimentellen Untersuchungen zeigte, dass tiefgefrorene Transplantate deutlich geringere immunologische Reaktionen auslösen. Die Qualität und biologische Aktivität dieses Knochens ist jedoch wesentlich schlechter als bei autogenem Material. Er ist aber in nahezu unbegrenzter Menge vorhanden und jede größere unfallchirurgische Klinik unterhielt ihre eigene Knochenbank. Durch die Zunahme der Bedeutung lebensgefährlicher viraler Infektionskrankheiten wie Hepatitis $C$ und AIDS mußte der Umgang mit allogenem Material wesentlich kritischer erfolgen als dies in den 70er und 80er Jahren der Fall war.

Nach Bekanntwerden der ersten durch Knochen übertragenen HIV-Infektion aus dem Jahre 1988 [1] kamen 1990 die ersten Richtlinien zum Führen einer Knochenbank vom wissenschaftlichen Beirat der Bundesärztekammer heraus [14]. Diese Richtlinien orientierten sich im Wesentlichen an den „bone bank procedures" aus den USA und wurden im August 1996 novelliert [15]. Die Einhaltung dieser vorgeschlagenen Maßnahmen bietet eine ausreichende Sicherheit für den betroffenen Patienten [10], sie führt allerdings auch dazu, dass Knochenbanken aufgrund der notwendigen Logistik und Infrastruktur sowie der entstehenden Kosten nur noch von einigen regionalen bzw. überregionalen Zentren vorgehalten werden können.

Aufgrund der hier geschilderten Problematik ist es verständlich, dass in den letz- 

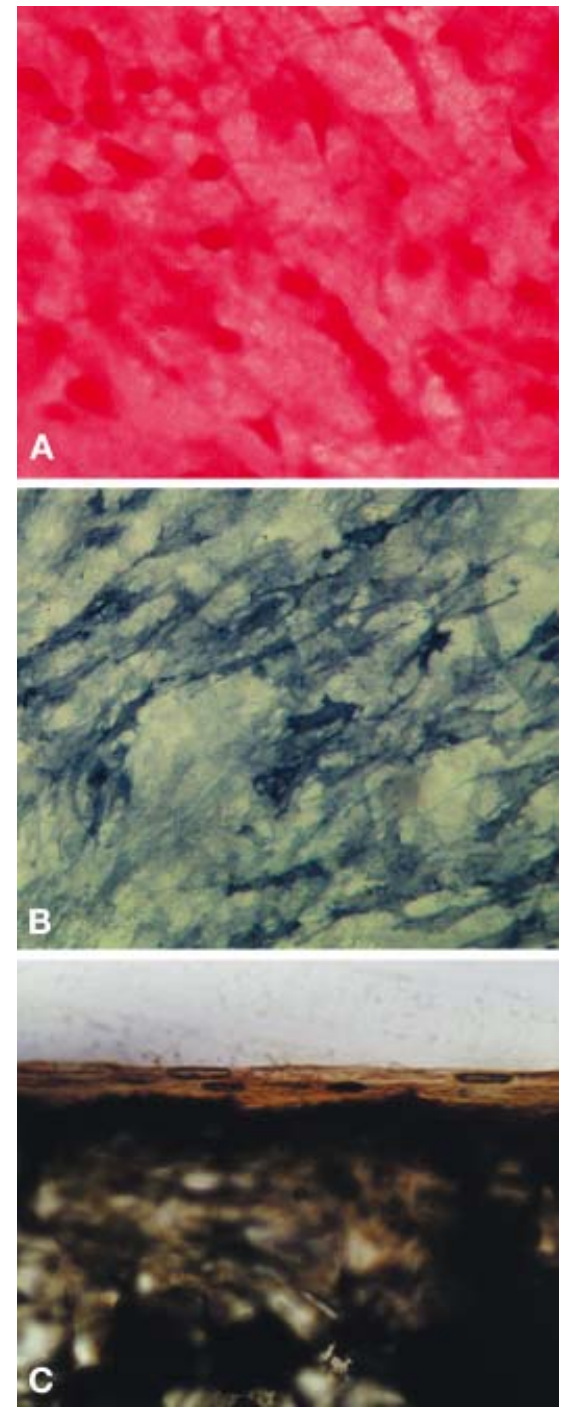

Abb.1 Osteoblastenspezifischer Biokompatibilitätsnachweis: In Anwesenheit eines Prüfkörpers werden authentische Osteoblasten kultiviert. Neben regelmäßigen Analysen des $\mathrm{pH}$-Wertes werden Syntheseprodukte des Osteoblasten zu definierten Zeitpunkten bestimmt. A: Kollagennachweis (rot) mittels van Gieson-Färbung. B: alkalische Phosphatase (blau). C: Osteoblasten-PrüfkörperInteraktion: Mehrschichtiger OsteoblastenLayer grenzschichtfrei nach 4 Wochen in Kultur auf einer Glaskeramik (GB9N, Fa. Biovision, Ilmenau).

ten Jahren die Suche nach geeigneten Knochenersatzmaterialien intensiviert wurde.

Unter dem Begriff Knochenersatzmaterialien werden Substanzen subsumiert, die in der Lage sein sollen, für alle Indikationen bei denen autogener Knochen transplantiert werden soll, diesen als Transplantatmaterial zu ersetzen.

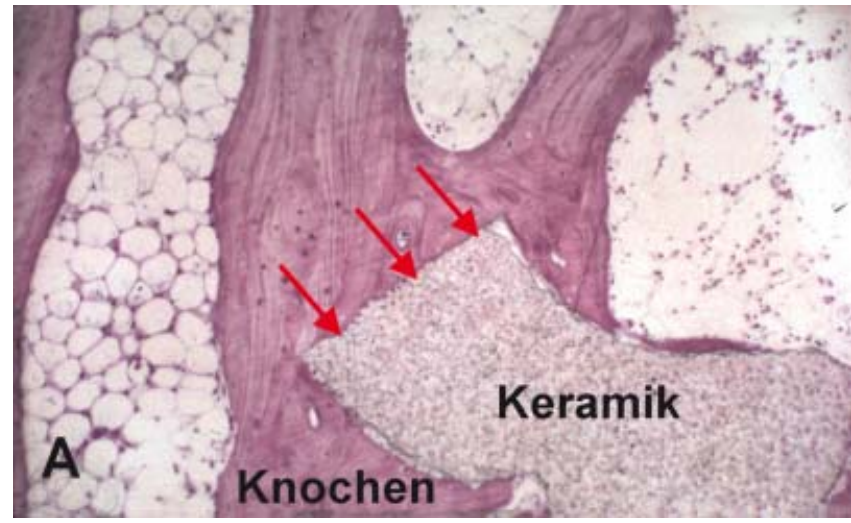

Abb. 2 Nachweis der grenzschichtfreien Integration $(\rightarrow)$ einer Hydroxylapatit-Keramik nach Implantation in einen metaphysären Defekt. A: Hämatoxylin-Eosin-Färbung. B: Polarisationsmikroskopie: Darstellung des senkrechten Auftreffens der Kollagenfasern auf der Oberfläche der

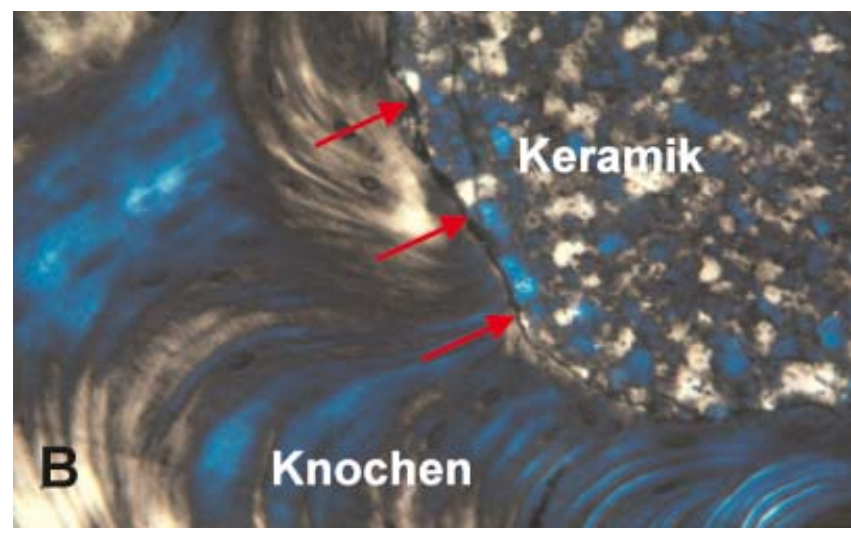
Keramik.

\section{Anforderungen}

\section{Grundsätzliche Anforderungen}

Grundsätzliche Anforderungen an ein Knochenersatzmittel ergeben sich aus Aufgaben, die dieses Material im lebenden Gewebe erfüllen soll:

Die Substanz darf daher keine Toxizität, keine Kanzerogenität, keine Teratogenität aufweisen, und sie soll keine systemischen unerwünschten Wirkungen haben. Stattdessen soll sie eine gute, möglichst osteoblastenspezifische Biokompatibilität besitzen (Abb.1).

Knochenersatzmittel sollten weiterhin eine hohe Bioaktivität besitzen, d.h. in einen Stoffaustausch mit Wirtszellen und Lagergewebe treten. Eine solche Bioaktivität ist neben strukturellen Anforderungen eine mögliche Bedingung für eine osteokonduktive Wirkung und unabdingbare Voraussetzung für eine osteostimulative oder sogar osteoinduktive Wirkung. Biomaterialien zum Knochenersatz sollten eine Substratwirkung besitzen, d.h. die Abbauprodukte können für den knöchernen Wiederaufbau am Implantationsort verwendet werden.
Knochenersatzmaterialien sollten möglichst die Freisetzung und/oder Aktivierung bestimmter den Knochenstoffwechsel positiv beeinflussender Mediatoren provozieren und im Idealfall auch als Trägermaterial für bestimmte Substanzen wie beispielsweise BMPs oder Antibiotika fungieren können.

Knochenersatzmaterialien müssen eine substanzielle Platzhalterfunktion erfüllen, um die Bildung und das Einwachsen von lockerem Granulationsgewebe oder Narbengewebe anstelle von Knochen zu verhindern. Mit ihrer Platzhalterfunktion sollten die Materialien eine hohe Primärstabilität verbinden, um mechanische Anforderungen zu erfüllen. Knochen hat als Stützgewebe im wesentlichen mechanische Aufgaben, die passager oder auf Dauer zu übernehmen sind.

Bei Materialien ohne ausreichende Festigkeit muss die Osteosynthese oder eine andere externe Fixierung die geforderte Stabilität bis zum knöchernen Durchbau sicherstellen [7].

Ideale Biomaterialien zum Knochenersatz erlauben das Aufwachsen von vitalem Knochen auf ihrer Oberfläche: Osteoblasten können Matrixbestandteile auf- 
bringen und damit die knöcherne Integration des Materials fördern (Abb.2).

Das ideale Knochenersatzmittel sollte teilweise oder vollständig biologisch abbaubar sein und dann von vitalem ortständigen Knochen ersetzt werden können.

Die Sterilisation, Lagerung und intraoperative Bearbeitung muss einfach, die Applikation sicher und reproduzierbar möglich sein. In der derzeitigen Situation des Gesundheitswesens darf auch der ökonomische Aspekt nicht vernachlässigt werden: Wenn allerdings ein Biomaterial alle genannten Forderungen erfüllt und damit die Verwendung autogenen Knochenmaterials in vielen Fällen vermeidbar macht, wird es auf jeden Fall auch kostengünstiger sein als die Erweiterung der Operation mit den bekannten möglichen Komplikationen.

\section{Klinische Anforderungen an Knochenersatzmaterialien}

Das ideale Knochenersatzmaterial besteht aus einer Substanz oder einem Substanzgemisch, das eine schnelle intensive Knochenneubildung am gewünschten Ort fördert.

Mit seiner eigenen Struktur und dem neu entstandenen Knochen erfüllt es die mechanischen Anforderungen der jeweiligen Skelettregion. Nach erreichtem knöchernen Durchbau kann es im Rahmen der Umbauvorgänge durch körpereigenen Knochen ersetzt werden, ohne dass es durch seinen Abbau zu mechanischen Schwächen oder zu systemisch bedenklichen Reaktionen kommt. Dieses ideale Material existiert bisher noch nicht. Einzelaspekte der Anforderungen werden bereits zuverlässig erfüllt, besonders aber in einer echten Induktionswirkung auf die Osteoneogenese und in einem ungestörten Remodeling und Ersatz des Biomaterials durch Knochen verbleiben noch viele Wünsche offen.

In Gelenknähe und speziell an der unteren Extremität sind hohe Anforderungen an die mechanischen Qualitäten des Knochenregenerates und des Knochenersatzmaterials zu stellen. Gelenknahe Implantationen werden im Wesentlichen auf Druck belastet. Diese Anforderung wird von einigen auf dem Markt befindlichen keramischen und verschiedene Composite-Materialien in Grenzen erfüllt. Nicht resorbierbare Kalziumsphosphat-Keramiken (z.B. Endobon ${ }^{\circledR}$, Merck Biomate-

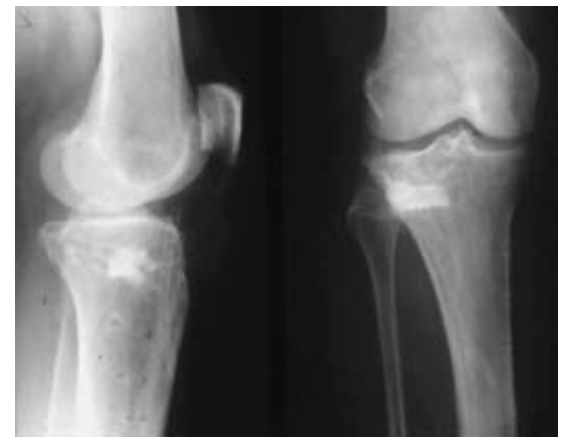

Abb. 3 Auch vier Jahre nach der Implantation wird der Endobon ${ }^{\circledR}$-Block intensiv kontrastgebend in der konventionellen Röntgenaufnahme abgebildet.

rialien) haben unstrittig große Erfolge bei der Defektfüllung großer vor allem spongiöser Defekte vorzuweisen. Kritische Autoren diskutieren vor allem die Problematik des lebenslangen Verbleibs dieser Stoffe bezüglich ihrer LangzeitBiokompatibilität. Tatsächlich stechen die hoch röntgendichten Implantate noch Jahre nach dem Einbau bei jeder Untersuchung ins Auge (Abb. 3). Unerwünschte Reaktionen konnten bisher allerdings nicht nachgewiesen werden.

Biologisch handelt es sich gelenknah (Tibiakopf, Pilon tibiale) um ersatzstarke knöcherne Lager, in denen schon osteokonduktive Eigenschaften zu zuverlässiger Defektüberbrückung führen.

Problematisch sind und bleiben Knochendefekte im Schaftbereich langer
Röhrenknochen, wo Rotations- und Biegebeanspruchung die Druckbelastung überlagern. Hier sind keramische Werkstoffe aufgrund fehlender Biegestabilität nicht einsetzbar. Realistischerweise können diese Problemdefekte mittels Knochenersatzmittel nur in aussichtslosen Tumorsituationen durch die Implantation eines metallischen Segmentersatzes therapiert werden. In allen anderen Fällen konkurrieren noch immer operative Verfahren wie die Callusdistraktion/Segmenttransport oder die freie Knochensegmenttransplantation (Abb.4).

An der oberen Extremität finden sich mechanisch geringer beanspruchte Regionen mit Knochendefekten, die mit Knochenersatzmaterial gefüllt werden müssen. Speziell metaphysäre Implantationen können hier mit Materialien durchgeführt werden, die mit geringerer Primärstabilität den knöcheren Durchbau ermöglichen und später - zumindest teilweise - abgebaut werden. $\mathrm{Zu}$ diesen Stoffen zählen die neuen Kalziumphosphatzemente Norian SRS ${ }^{\circledR}$ (Mathys) und Biobon ${ }^{\circledR}$ (Merck Biomaterialen), die sich graduell in ihrer Zusammensetzung unterscheiden. Es handelt sich jeweils um im OP aus Pulver und physiologischer Flüssigkeit angemischte pastenartige Verbindungen, die in situ aushärten und dann als Platzhalter und möglicherweise als bioaktiver Stimulus für die Osteoneogenese wirken. Bereits kurz nach der Implantation kommt es zu ersten Degradationsvorgängen und nach etwa $1 / 2$ Jahr sind bereits nennenswerte Mengen ra-
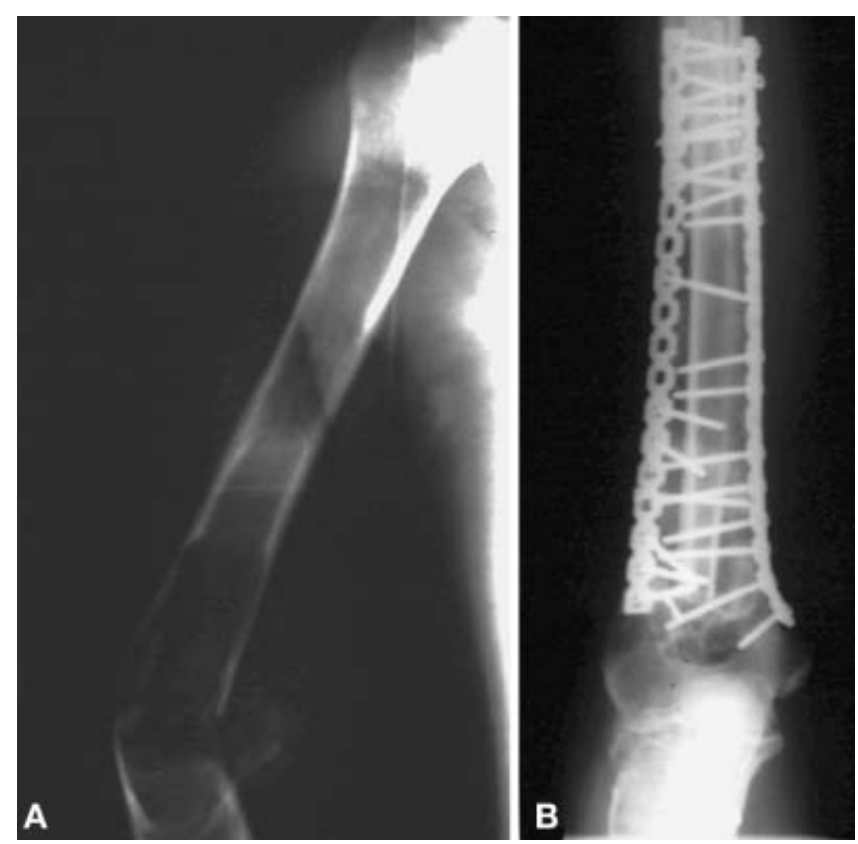

Abb. 4 Pathologische Humerusschaftfraktur bei fibröser Dysplasie. Nach radikaler Tumorausräumung wurde ein $21 \mathrm{~cm}$ langes Fibulatransplantat in den entstandenen Defekt eingebolzt und mittels Plattenosteosynthese stabilisiert. A: Unfallbild. B: Kontrolle nach zwei Jahren. 

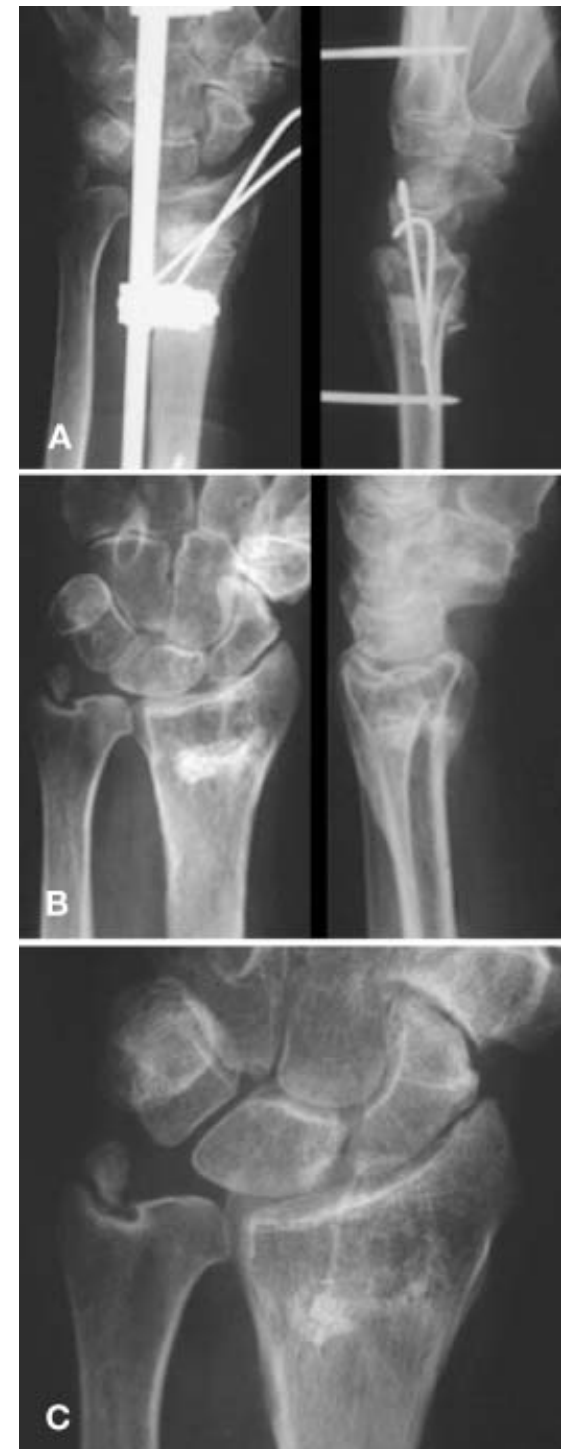

Abb.5 Operative Stabilisierung einer distalen Radiusfraktur mittels Fixateur externe und Kirschner-Draht-Osteosynthese bei einer 69 jährigen Patientin. Der knöcherne Defekt im Bereich der dorsalen Trümmerzone wurde mit Biobon ${ }^{\circledR}$ aufgefüllt. Es zeigt sich eine zunehmende Strahlentransparenz des eingebrachten Kalziumphosphatzementes im Verlauf eines Jahres, als Ausdruck der Biodegradation der Substanz. A: Erster postoperativer Tag. B: Kontrolle nach 6 Monaten. C: Kontrolle nach einem Jahr.

diologisch nicht mehr nachweisbar (Abb.5). Für Norian SRS ${ }^{\circledR}$ ist experimentell eine höhere Primärstabilität nachgewiesen.

Aufgrund der exzellenten Bioverträglichkeit und des schnellen Abbaus in den Weichteilen oder nach akzidenteller intraartikulärer Applikation hat sich ihr Einsatz - in Kombination mit einer stabi-

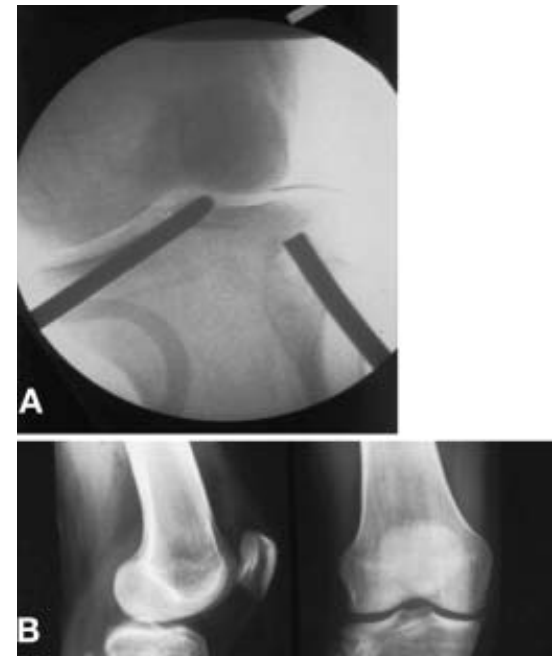

Abb. 6 Arthroskopisch kontrollierte Anhebung der lateralen Gelenkfläche nach Tibiakopf-Impressionsfraktur bei einer 26 jährigen Patientin. Der entstandene Knochensubstanzdefekt wurde mit Biobon ${ }^{\circledR}$ aufgefüllt. A: Intraoperatives Durchleuchtungsbild. B: Postoperative Kontrolle mit dem kontrastgebenden Calziumphosphatzement. Auf eine zusätzliche osteosynthetische Stabilisierung wurde in diesem Fall verzichtet.

len Osteosynthese - auch bei Gelenkfrakturen an der unteren Extremität bewährt. Vor allem für arthroskopisch gestützte Gelenkflächenrekonstruktionen nach Tibiakopffrakturen stellen sie nicht zuletzt dank der Möglichkeit der minimalinvasiven Spritzenapplikation eine wirkliche Alternative zur Spongiosaplastik oder keramischen Knochenersatzmitteln dar (Abb. 6).

Composite-Werkstoffe sind Verbindungen unterschiedlicher Substanzen, die als Knochenersatzmittel eingesetzt werden mit dem Ziel die biologischen und/ oder die mechanischen Eigenschaften der Einzelsubstanzen durch die Kombination zu verbessern.

Die seit vielen Jahren im klinischen Einsatz befindlichen Hydroxylapatit-Keramiken werden teilweise vor der Implantation mit autogenem Knochenmark oder Spongiosa augmentiert, um die biologische Wirksamkeit im Knochen zu verbessern. Im Grunde wird so ein CompositeWerkstoff hergestellt, bei dem das Trägermaterial Hydroxylapatit mit körpereigenen Faktoren bzw. Knochenzellen kombiniert wird.

Vor allem die unbefriedigenden mechanischen Eigenschaften der bekannten
Kalziumcarbonat/-phosphat-Verbindungen, haben dazu geführt, dass stabilere Materialien für den Knochenersatz entwickelt werden.

Resorbierbare Kunststoffe wie Polylactid und Polyglycolid werden als resorbierbare Implantate klinisch eingesetzt. Vorteil dieser Polyesterverbindungen ist ihre hohe mechanische Belastbarkeit, als nachteilig erweist sich die Tatsache, dass lokale Gewebereaktionen und Osteolysen auftreten können [12].

Durch die Kombination von Polylactid und Polyglycolid können sowohl die mechanischen Eigenschaften als auch die Degradationszeit beeinflusst werden. Um das Problem der lokalen Übersäuerung zu verringern und die Gewebeakzeptanz zu erhöhen, gibt es einige experimentelle Ansätze Polyester sowohl mit verschiedenen Carbonatapatitphasen zu kombinieren [6] als auch mit $\alpha$-TCP oder verschiedenen Glaskeramiken [2].

Aufgrund ihrer Biodegradibilität würden sich derartige Materialien auch als Trägersubstanzen für bestimmte Medikamente (z.B. Antibiotika, Zytostatika) oder Wachstumsfaktoren eignen. Da für die Herstellung von Formkörpern auf der Grundlage von Polyestern in der Regel hohe Temperaturen notwendig sind, ist die Verwendung von Proteinen in derartigen Composite-Werkstoffen zur Zeit technisch nur schwer zu realisieren.

Hingegen wurden bereits vielversprechende präklinische Ansätze bezüglich der Kombination von rekombinantem BMP-2 und einem Kalziumphosphatzement publiziert [4]. In dieser Studie wurde gezeigt, dass ein „critical size defect“ im Kaninchenradius, der durch den Einsatz von rhBMP-2 und $\alpha$-BSM überbrückt wurde, zur schnelleren knöchernen Ausheilung führte, als bei der alleinigen Applikation von $\alpha$-BSM. Durch die Freisetzung des Wachstumsfaktors wird wahrscheinlich die lokale Osteoinduktion - d. h. Zellteilung von definierten Stammzellen, Differenzierung der Knochenzellen und spezifische Matrixproduktion der Osteoblasten - unterstützt und führt so möglicherweise zu einer Beschleunigung der Knochenneubildung erreicht.

Potenziell sind Kombinationen von Substanzen aller Knochenersatzmittel-Klassen denkbar. 


\section{Ausblick}

Als eine erfolgversprechende Maßnahme bietet sich für die Zukunft neben der Implantation von Knochenersatzmaterialien unter Zusatz von Wachstumsfaktoren die die In-vitro-Züchtung von differenziertem Knochengewebe an. Hierzu werden differenzierte Knochenzellen oder mesenchymale Stammzellen aus einer minimalinvasiven Knochenmarkpunktion verwendet. Sie werden von Matrixproteinen befreit und in einem Proliferationsschritt massiv vermehrt. Anschließend werden diese Zellen in einem weiterhin kontrollierten Redifferenzierungsprogramm im Labor zur Osteoneogenese angeregt. Hierbei können Biomaterialien in Form von Polymer-Vliesen oder porösen Kalziumphosphat-Verbindungen oder Composite Hilfestellung leisten, indem sie den Zellen bioaktive Oberflächen anbieten, auf denen sie sich ausdifferenzieren und Matrixproteine abscheiden. Die Vorarbeiten an den Knochenersatzmaterialien können vollständig in die Überlegungen zur Nutzbarkeit der Biomaterialien für das Tissue Engineering eingebracht werden.

Problematisch bleibt die echte Gewebezüchtung von Knochen aufgrund seiner komplexen Vaskularität: Es können bisher nur gefäßfrei zu ernährende Gewebestücke erzeugt und transplantiert werden. Kokulturen mit Gefäßsprossen haben auch bei anderen Organsystemen wie Leber oder Muskel bisher keine durchschlagenden Erfolge erzielt [3].

\section{Schlussfolgerung}

Die momentan zur Verfügung stehenden Substanzen entsprechen sicherlich noch nicht der Idealvorstellung, die wir von derartigen Materialien haben, aber sie erlauben uns, bei vielen Indikationen vor allem im metaphysären Bereich auf die am Entnahmeort komplikationsträchtige autogene Spongiosaplastik zu verzichten. Inwieweit in Zukunft der Einsatz von ,intelligenten“ Knochenersatzmitteln und Tissue Engineering Bedeutung erlangen werden, hängt nicht zuletzt von der Leistungsfähigkeit unseres Gesundheitswesens ab.

\section{Literatur}

${ }^{1}$ Center for Disease Control. Transmission of HIV through bone Transplantation: Case report and public health recommendations. 1988; 37: 597 - 599

${ }^{2}$ Claes L, Wolf S, Ignatius A. Mechanische Eigenschaften von biodegradablen Polymeren und Keramiken. OP-Journal 1998; 14: $230-233$

${ }^{3}$ Isogai N, Landis W, Kim TH, Gerstenfeld LC, Upton J, Vacanti JP. Formation of phalanges and small joints by tissue-engineering. J Bone Joint Surg 1999; 81 A: 306-316

${ }^{4}$ Lee DD, Tofighi A, Aiolova M, Chakravarthy P, Catalano A, Majahad A, Knaack D. $\alpha-$ BSM $^{\circledR}$ : A biomimetic bone substitute and drug delivery vehicle. Clin Orthop 1999; 367 (Suppl.): S396 - S405

${ }^{5}$ Liebendörfer A, Tröster S. Hydroxylapatitkeramik im klinischen Einsatz. Histologische Befunde von 23 Patienten. Unfallchirurgie 1997; 23: 60-68

${ }^{6}$ Linhart W, Peters F, Lehmann W, Schwarz K, Schilling AF, Amling M, Rueger JM, Epple M. Biologically and chemically optimized composites of carbonated apatite and polyglycolide as bone substitution materials. J Biomed Mat Res, im Druck 2000

${ }^{7}$ Rueger JM. Knochenersatzmittel. In: J. Rehn, L. Schweiberer, H. Tscherne, (Hrsg.) Hefte zur Unfallheilkunde 213 Springer Verlag Berlin, Heidelberg 1992

${ }^{8}$ Sailer R, Gabl M, Lutz M, Seykora P, Pechlaner S. Integration einer porösen Hydroxylapatitkeramik am distalen Radius älterer Patienten. Unfallchirurg 1999; 102: $531-534$

${ }^{9}$ Schaller P, Geldmacher J, Freiberger N, Flügel M. Die Auffütterung kleiner Knochenhöhlen mit Knochenkeramik (Hydroxylapatit). Handchir Mikrochir Plast Chir 1993; 25: 184- 190
10 Sommerfeldt DW, Linhart W, Schmandra TC, Konold P, Rueger JM. Die Knochenbank: Richtlinien - Probleme - Anwendung. Unfallchirurgie 1998; 24: 236-244

${ }^{11}$ Van Heest A, Swiotkowski M. Bone-graft substitutes. Lancet 1999; 353 (suppl I): 28-29

12 Weiler A, Helling H, Kirch U, Zirbes T, Rehm KE. Forign-body reactions and the course of osteolysis after polyglycolide implants for fracture fixation - Experimentel study in sheep. J Bone Joint Surg 1996; 78 B: 369 376

${ }^{13}$ Wippermann BW, Schratt HE, Steeg S, Tscherne H. Komplikationen der Spongiosaentnahme am Beckenkamm. Eine retrospektive Analyse von 1191 Fällen. Chirurg 1997; 68: $1286-1291$

${ }^{14}$ Wissenschaftlicher Beirat der Bundesärztekammer. Richtlinien zum Führen einer Knochenbank. Dtsch Ärztebl 1990; 87: 39-42

${ }^{15}$ Wissenschaftlicher Beirat der Bundesärztekammer. Richtlinien zum Führen einer Knochenbank. Dtsch Ärztebl 1996; 93: 34-35

\section{Dr. med. W. Linhart}

Oberarzt

Priv.-Doz. Dr. med N. M. Meenen

Ltd. Oberarzt

Prof. Dr. med. J. M. Rueger

Ärztlicher Direktor

Unfall- u. Wiederherstellungschirurgie Universitätsklinikum HamburgEppendorf Martinistraße 52

20246 Hamburg 